\title{
Analyse de formules employées par le gouvernement et de leurs stratégies de cadrage sous-jacentes pendant le printemps érable québécois de 2012
}

Analysis of formulas used by the government and their underlying framing strategies during the 2012 Maple Spring in Quebec

\section{Laurence Pelletier et Marie-Josée Goulet}

\section{(2) OpenEdition}

\section{Journals}

Édition électronique

URL : http://journals.openedition.org/aad/2688

DOI : 10.4000/aad.2688

ISSN : 1565-8961

Éditeur

Université de Tel-Aviv

Référence électronique

Laurence Pelletier et Marie-Josée Goulet, « Analyse de formules employées par le gouvernement et de leurs stratégies de cadrage sous-jacentes pendant le printemps érable québécois de 2012 », Argumentation et Analyse du Discours [En ligne], 21 | 2018, mis en ligne le 15 octobre 2018, consulté le 23 septembre 2019. URL : http://journals.openedition.org/aad/2688 ; DOI : 10.4000/aad.2688

Ce document a été généré automatiquement le 23 septembre 2019.

\section{(1) $\$ \odot$}

Argumentation \& analyse du discours est mis à disposition selon les termes de la licence Creative Commons Attribution - Pas d'Utilisation Commerciale - Pas de Modification 4.0 International. 


\section{Analyse de formules employées par le gouvernement et de leurs stratégies de cadrage sous-jacentes pendant le printemps érable québécois de 2012}

Analysis of formulas used by the government and their underlying framing strategies during the 2012 Maple Spring in Quebec

Laurence Pelletier et Marie-Josée Goulet

\section{Retour sur le printemps érable québécois de 2012}

1 Nous revenons dans cet article sur un événement qui a marqué le Québec en 2012 : le printemps érable. Le catalyseur de cette crise sociopolitique remonte à mars 2011, lorsque le gouvernement annonce une hausse de $75 \%$ des frais de scolarité universitaire. Se déclenche alors un mouvement de grève générale illimitée de plus de 300000 étudiants postsecondaires à son apogée (Ancelovici et Dupuis-Déri 2014, BéginCaouette et Jones 2014, Bernard-Barbeau 2015 ; Bonenfant et al. 2013, Poirier St-Pierre et Éthier 2013). Sur le plan des acteurs, la polarisation du conflit entre le gouvernement libéral - au premier chef le premier ministre Jean Charest, les ministres des Finances, Raymond Bachand, et de l'Éducation, Line Beauchamp, puis Michelle Courchesne - et les principales associations étudiantes postsecondaires - la Fédération étudiante collégiale du Québec (FECQ), la Fédération étudiante universitaire du Québec (FEUQ) et la Coalition large de l'Association pour une solidarité syndicale étudiante (CLASSÉ) - a largement été rapportée (en plus des auteurs mentionnés plus haut: Carbonneau et Desrochers 2013, Frappier et al. 2012, Sorochan 2012). Selon Gauthier (2016), le mouvement s'est généralisé en une contestation du gouvernement en place et en une dénonciation du système capitaliste néolibéral. C'est l'élection du gouvernement de 
Pauline Marois le 4 septembre 2012 qui marque la fin du printemps érable (Ancelovici et Dupuis 2014, Bernard-Barbeau 2015, Frappier 2012, Julien 2012, Poirier et Éthier 2013).

2 Comme l'explique Orkibi (2015), le discours est généralement très marqué autour des questions qui divisent la société. Parmi les rares études scientifiques sur le printemps érable, celles qui ont traité du discours des étudiants rapportent des divergences dans les stratégies discursives de ces derniers (Bernard-Barbeau 2015, Lacroix et al. 2014). À l'inverse, les études portant sur le discours du gouvernement soulignent l'homogénéité de sa stratégie : plusieurs auteurs ont noté la présence d'expressions inspirées d'une idéologie ou rhétorique «libérale individualiste» (Ancelovici et Dupuis 2014), «néolibérale» (Frappier 2012), « paternaliste» (Bonenfant 2013) ou encore d'une logique de "calcul rationnel» (Lynes 2012). Or, la démonstration empirique d'une stratégie discursive appuyée sur des expressions créées et employées par le gouvernement reste à faire; ces dernières sont mentionnées de façon anecdotique, sans support quantitatif quant à leur fréquence ou leur intensité. Cela peut s'expliquer par le fait que la plupart des textes pragmatiques et universitaires ayant été publiés au sujet du printemps érable sont des analyses critiques ou des essais (Ancelovici et Dupuis 2014). À la lumière de ces constats, il nous apparaissait pertinent d'étudier de manière systématique les formules utilisées par le gouvernement pendant le printemps érable. Au moyen d'une analyse de contenu de 100 articles de presse, nous avons étudié 152 formules employées par le gouvernement pendant le printemps érable. Dans cette analyse, nous avons pris en compte la distribution des formules dans le temps, les entités qu'elles ont ciblées, les locuteurs les ayant employées et les stratégies de cadrage qu'elles sous-entendent. Par cadrage nous entendons des procédés sémantiques et linguistiques précis visant à manipuler la perception qu'ont les individus d'une situation ou d'un événement (Entman 1993), en l'occurrence, le printemps érable. Nous reviendrons plus en détail sur ce concept à la section 3 portant sur le cadre conceptuel.

\section{2. Études antérieures sur le cadrage pendant le printemps érable}

3 L'étude de Millette (2013), basée sur des entretiens en profondeur ${ }^{1}$, ainsi que sur une recherche documentaire de contenus médiatiques dans les bases de données Eureka et PubliQc, a révélé la présence de quatre cadres pour éclairer les «dynamiques de tensions entourant la question du recours aux relations publiques et des relations aux médias » (66) :

- la personnalisation du conflit

- le contre-mouvement

- le « boycottage » et la « grève »

- la violence et l'intimidation

4 Au sujet du premier cadre, l'auteure en a attribué la création aux médias de masse du Québec (notamment le Journal de Montréal et le groupe Quebecor) qui, selon elle, ont cherché à diviser l'opinion publique en présentant le conflit sous forme de duels entre personnalités publiques. Par exemple, certains médias mettaient l'accent sur le 
parcours des leaders étudiants comme Gabriel Nadeau-Dubois (CLASSÉ), Léo BureauBlouin (FECQ) ou Martine Desjardins (FEUQ).

Millette (2013) a également évoqué la stratégie menée par les médias pour intéresser le public à la question des frais de scolarité : la polarisation du conflit entre les «carrés verts " - représentés par le Mouvement des étudiants socialement responsables du Québec (MESRQ) et la Commission jeunesse du Parti libéral du Québec (CJPLQ) -, et les " carrés rouges ", représentés par les quatre principales associations étudiantes, soient la CLASSÉ, la FEUQ, la FECQ et la Table de concertation étudiante du Québec (TaCEQ).

En ce qui a trait aux cadres du "boycottage » et de la "grève ", il s'agissait, toujours selon Millette, d'un enjeu stratégique profitant aux deux camps impliqués dans le conflit :

D’un côté, la "grève " étudiante tire sa force de son caractère collectif et, par conséquent, de sa capacité à enrayer efficacement le fonctionnement des universités tandis que, de l'autre côté, le «boycottage » suppose une action individuelle, donc, une responsabilité tout aussi individuelle face aux conséquences de cette action (2013: 70).

Comme l'affirme Gauthier (2016), ce conflit de nomination entre "grève » et «boycott » a obscurci le débat sur l'augmentation des droits de scolarité.

Enfin, le cadre de la violence et de l'intimidation, tout comme le cadre précédent, constituait la trame de fond du discours utilisé tant par le gouvernement (dénonçant les actions des manifestants dans les bureaux de la ministre de l'Éducation) que par le mouvement étudiant (dénonçant la répression policière). Exacerbé par les représentations médiatiques violentes des manifestations, le cadre de la violence aurait toutefois été plus profitable au gouvernement, permettant au Parti libéral du Québec (PLQ) de se positionner comme «[...] un tenant de la ligne dure et du respect des autorités politiques, judiciaires et policières » (Millette 2013: 82).

Pour leur part, Carbonneau et Desrochers (2013) ont voulu mettre l'accent sur le traitement du printemps érable par les médias, afin de déterminer si ceux-ci avaient contribué à la polarisation de l'opinion publique. À partir d'un corpus de 185 articles parus dans cinqjournaux québécois deuxjours après les deux plus grandes manifestations de rue, les auteurs ont procédé à une analyse du discours des médias. Leur grille d'analyse prévoyait une classification des articles selon :

- le type d'énonciation: objectivisé (comptes-rendus, reportages et enquêtes) ou subjectivisé (éditoriaux et chroniques)

- la position adoptée : en faveur des étudiants, en faveur du gouvernement ou propos nuancés

- la thématique : anecdotique, si l'article portait surtout sur les débordements, ou analytique, s'il portait plutôt sur les enjeux de fond du conflit

- la dimension cognitive de la manipulation (Breton 1997) : recadrage abusif, si la stratégie consistait à employer des mots piégés ou chargés péjorativement, ou amalgame cognitif, si les leviers suivants étaient plutôt employés :

1) les leviers de vertu, dénotant une association avec des symboles tenus pour bons, par exemple « démocratie », « légitimité » et « culture civique », ou « discipline » dans le cas de la grève étudiante,

2) les leviers poison, apparents lorsqu'une idée est associée avec des symboles tenus pour mauvais comme " guerre » et « mort » ou dans le cas de la grève, « zone de guerre ", 
3) les leviers d'autorité qui s'appuient sur l'expertise, la notoriété ou l'autorité de personnes bien connues et d'institutions pour faire accepter une idée,

4) les leviers de conformisation, qui font appel à la solidarité et à l'identification du lecteur avec une communauté humaine en particulier, comme le dénote le recours à la « majorité silencieuse » (Carbonneau et Desrochers $2013: 9$ ).

$10 \mathrm{Au}$ terme de leur analyse, Carbonneau et Desrochers concluent que la couverture médiatique aurait favorisé une thématique anecdotique plutôt qu'analytique. Moins de $20 \%$ des articles classifiés affichaient une présence de cadres, ce qui, selon les auteurs, permettait de réfuter leur hypothèse initiale qui attribuait, de façon générale, « un rôle aux médias dans la polarisation sociale » (17). En contrepartie, les auteurs soulignent que The Gazette et le Journal de Montréal s'étaient démarqués des autres journaux, le contenu de leurs articles semblant illustrer une volonté d'influencer le lecteur en défaveur du mouvement étudiant.

11 Tout compte fait, les études de Millette et de Carbonneau et Desrochers présentent des méthodologies et des résultats divergents. D'une part, une recherche documentaire et des entrevues avec des membres d'associations étudiantes ont révélé la présence de quatre cadres dans le discours des médias, du gouvernement et du mouvement étudiant. D'autre part, une analyse de contenu de 185 articles a mené au rejet de l'hypothèse de la présence de cadres dans la couverture médiatique de deux manifestations étudiantes. Quant à nous, nous allons recourir à la formule comme unité d'analyse afin de produire des données quantitatives sur la stratégie discursive du gouvernement pendant le printemps érable.

12 Nos questions de recherche sont les suivantes:

- Comment l'emploi de formules a-t-il fluctué sur le plan temporel pendant le printemps érable ? Peut-on observer des différences quant à l'utilisation de formules en fonction des mois de l'événement?

- En quoi les formules ont-elles été mises au service d'une rhétorique visant à convaincre ? À quelles stratégies de cadrage ces formules ont-elles fait appel ?

- Qui sont les locuteurs qui, en employant des formules, se sont prononcés au nom de la stratégie discursive du gouvernement ? Observe-t-on des différences quant à l'utilisation de formules en fonction des locuteurs?

- Sur le plan du contenu, quelles entités (groupes, individus) la stratégie discursive et les formules visent-elles?

\section{Cadre conceptuel}

13 Le discours comme foyer d'idéologies et outil de maintien du pouvoir constitue un objet d'étude privilégié en analyse critique du discours (Fairclough 2001, Van Dijk 1985). Selon cette perspective, le discours du gouvernement - en particulier dans un contexte de crise sociale comme le printemps érable - peut être perçu comme un outil lui permettant d'affirmer et de préserver sa position de pouvoir. Le printemps érable a été décrit par certains comme une "guerre des mots" opposant le gouvernement aux étudiants (Bonenfant 2013, Carbonneau et Desrochers 2013, Millette 2013, Poirier et Éthier 2013). Pendant cet événement, le gouvernement aurait tenté d'exercer son pouvoir et d'imposer son idéologie en «jouant sur les mots» (Bonenfant, Glinoer et Lapointe 2013:184), avec des «jeux de langage, en termes de retournement et détournement des expressions et des valeurs sémantiques qu'on leur attribue en 
contexte» (Collette 2013:93). Cette idée d'attribution de différentes valeurs sémantiques selon le contexte fait écho au concept de cadrage. Le cadrage a contribué à enrichir divers domaines et disciplines des sciences sociales: psychologie cognitive, linguistique et analyse du discours, communications et sciences politiques, entre autres (Benford et Snow 2000). Souvent associé au discours de l'action collective et aux mouvements sociaux (Orkibi 2015), le cadrage peut également être envisagé comme un moyen pour les institutions officielles d'exploiter le langage pour affirmer leur pouvoir (Fairclough 2001, Van Dijk 1985). Dans cette perspective, le discours du pouvoir est émaillé de procédés sémantiques et linguistiques précis-de cadres - visant à manipuler la perception qu'ont les individus et la société au sens large d'une situation ou d'un événement à travers la mise en exergue d'éléments saillants, d'affirmations, de jugements et d'interprétations venant appuyer la thèse défendue (Entman 1993). Sous quelles formes les cadres, ces procédés sémantiques et linguistiques, se manifestent-ils ? Le concept de formule s'agence au concept de cadrage et offre une piste de réponse : il s'agit d'un élément discursif s'imposant avec une "fonction de cadrage du débat" (Krieg-Planque 2009 : 100). La formule se définit comme « un ensemble de formulations qui, du fait de leurs emplois à un moment donné et dans un espace public donné, cristallisent des enjeux politiques et sociaux que ces expressions contribuent dans le même temps à construire » (7). Une formule peut constituer une unité lexicale simple ou complexe, une expression, une petite phrase ou «toute autre phrase enregistrée dans la mémoire collective (par exemple "Plus jamais ça!") ", et, toujours selon KriegPlanque, la formule a quatre propriétés constituantes (2009:64 et 63) :

1) présente un caractère figé,

2) s'inscrit dans une dimension discursive,

3) fonctionne comme un référent social,

4) comporte un aspect polémique.

14 À la lumière de ce qui précède, notre hypothèse de travail est la suivante: dans le contexte du printemps érable, le gouvernement a cherché à influencer l'opinion publique et à exercer son pouvoir par l'entremise d'un discours étayé sur des cadres se rapportant à une idéologie précise. En analysant le discours du gouvernement, perceptible par l'emploi de formules, nous cherchons à voir en quoi la "partie dominante, ici le gouvernement» (Collette $2013: 83$ ) se sert de cette unité discursive pour cadrer l'opinion publique et réaffirmer sa position de pouvoir.

15 Afin d'étudier comment le cadrage s'articule dans un contexte d'analyse de formules, nous avons fait appel à la typologie de Breton dans La parole manipulée (1997). Cette typologie offre des outils méthodologiques nous permettant de lier le concept de formule à celui de cadrage et d'analyser en profondeur la stratégie discursive du gouvernement. Comme l'ont proposé Carbonneau et Desrochers (2013), nous avons retenu les concepts liés à la manipulation cognitive, plus précisément au recadrage abusif et à l'amalgame cognitif. Le recadrage abusif consiste à employer un discours qui ordonne les faits de telle façon que la nouvelle image de la réalité entraîne la conviction de l'auditoire (Breton 1997 : 114). Cette refonte du sens des mots peut se traduire par l'emploi de mots piégés, des "vocables au contenu tendancieux» qui suscitent une indignation chez le public (115). À cet effet, Breton cite le vocable «terrorisme», qui sert à transférer l'indignation qu'il suscite sur ceux qui en sont qualifiés. Le recadrage abusif peut également se manifester par la naturalisation du réel, soit l'utilisation de termes «déresponsabilisants» (120) visant à faciliter l'assimilation des idées du 
locuteur par l'auditoire (par exemple la "tragédie vietnamienne» plutôt que la "guerre du Viêt-Nam»). Les rails mentaux constituent aussi une technique de recadrage abusif, qui se résume à employer des mots qui « contraignent à voir dans la réalité présentée seulement certains de ses aspects, ou encore des éléments qui n'y figurent pas normalement" (117). Les rails mentaux, comme l'indique leur nom, entraînent la pensée "sur une tout autre voie que l'évaluation rationnelle » (120), comme en témoigne l'exemple fourni par Breton : « délivrer des missiles ».

Dans l'amalgame cognitif, le discours sert à «rendre acceptable une opinion, sans discussion de son contenu, avec un élément extérieur, sans rapport immédiat avec cette opinion, mais considéré, lui, comme déjà accepté par l'auditoire » (126)². Breton s'inspire des travaux de Tchakhotine et de Clyde R. Miller³, lorsqu'il évoque quatre "leviers " d'amalgame cognitif: leviers de vertu, leviers poison, leviers d'autorité ou de témoignage, et leviers de conformisation. Les leviers de vertu tentent de faire accepter des idées "en les associant avec des mots ou des symboles tenus pour "bons", par exemple "démocratie", "liberté", “justice", "patrie" " (128). Les leviers poison suggèrent également une association d'idées avec des mots, mais ceux-ci sont chargés d'une connotation négative, par exemple guerre, mort, fascisme, etc. (ibid.). Les leviers d'autorité ou de témoignage évoquent l'expérience et l'autorité de personnes ou d'institutions connues (Dieu, la loi, etc. - ibid.). Enfin, les leviers de conformisation " font appel à la solidarité, à la pression des émotions ou des actions collectives » afin d'inviter l'auditoire à se rallier à ce que propose le locuteur : «L'union fait la force » en constitue un exemple (ibid.).

\section{Collecte et codification des formules}

Les formules que nous analysons sont issues d'articles de presse publiés entre le 17 mars 2011 et le 4 septembre 2012. Ces dates coïncident avec le début et la fin du printemps érable, selon plusieurs sources recensées ${ }^{4}$ (Asselin 2012, Bégin-Caouette et Jones 2014, Bernard-Barbeau 2015, Carbonneau et Desrochers 2013, Collette 2013, Lynes 2012, Sorochan 2012). Seuls les articles au type d'énonciation objectivisé ont été retenus, c'est-à-dire les comptes-rendus, les reportages et les enquêtes. Les articles proviennent de cinq quotidiens francophones de la région métropolitaine montréalaise : 24 heures, Journal de Montréal, Le Devoir, La Presse et Métro.

La première recherche ayant généré 4566 articles, un échantillon par quotas a été formé. Pour ce faire, nous avons pris comme point de départ le nombre d'articles publiés par mois de la période sur laquelle s'est déroulé le printemps érable. Cette information nous était accessible dans la base de données électronique regroupant les cinq quotidiens, Eureka. Puisque des quantités inégales d'articles selon le mois de publication avaient été observées à l'étape de collecte des données, nous avons choisi de contrôler cette variable pour limiter les biais. L'échantillon est constitué de 100 articles.

À partir des quatre propriétés constituantes de la formule de Krieg-Planque, nous avons établi une liste de critères de repérage des expressions qui sont potentiellement des formules :

- les expressions placées entre guillemets

- les expressions répétées au sein d'un article

- les expressions répétées au sein de plusieurs articles 
- les expressions complexes dont une unité est répétée au sein d'un même article

- les expressions dénotant une figure de style (ex. : métaphore ou analogie)

Au total, 152 formules différentes ont été extraites. Une fois la procédure établie, nous avons codifié l'ensemble des formules à l'aide d'une grille comportant les catégories suivantes: formule, critère de repérage, type de discours (citation ou propos rapportés), contexte (phrase contenant la formule), quotidien, date de publication, nom du fichier, locuteur (individu ou entité employant la formule), cible (individu ou entité visée par la formule) et stratégies de cadrage.

21 Afin d'analyser les stratégies de cadrage sous-jacentes aux formules, les catégories de Breton (1997) ont été opérationnalisées sous forme de questions. Ce processus est présenté à la Figure 1.

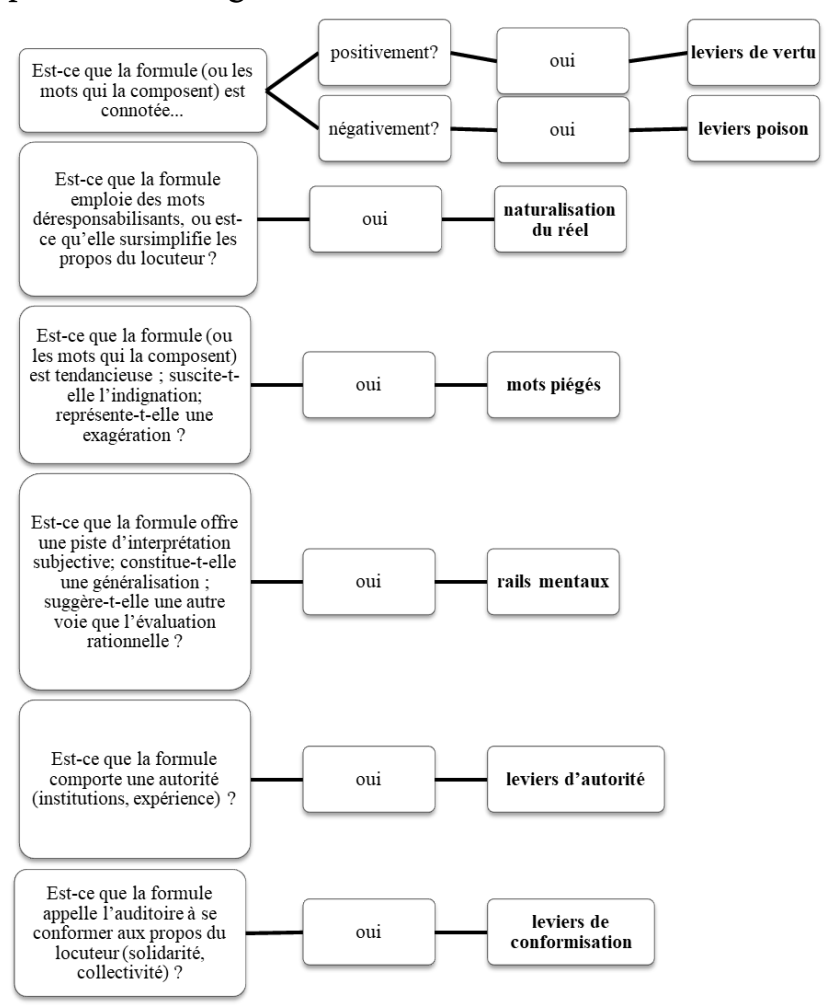

Figure 1 : Opérationnalisation des stratégies de cadrage de Breton

\section{Analyse des formules employées par le gouvernement}

\subsection{Mois d'apparition, locuteurs et cibles des formules}

La fréquence des formules employées varie selon le mois des événements, leur distribution dans le temps étant déterminée par la date de publication des articles. 


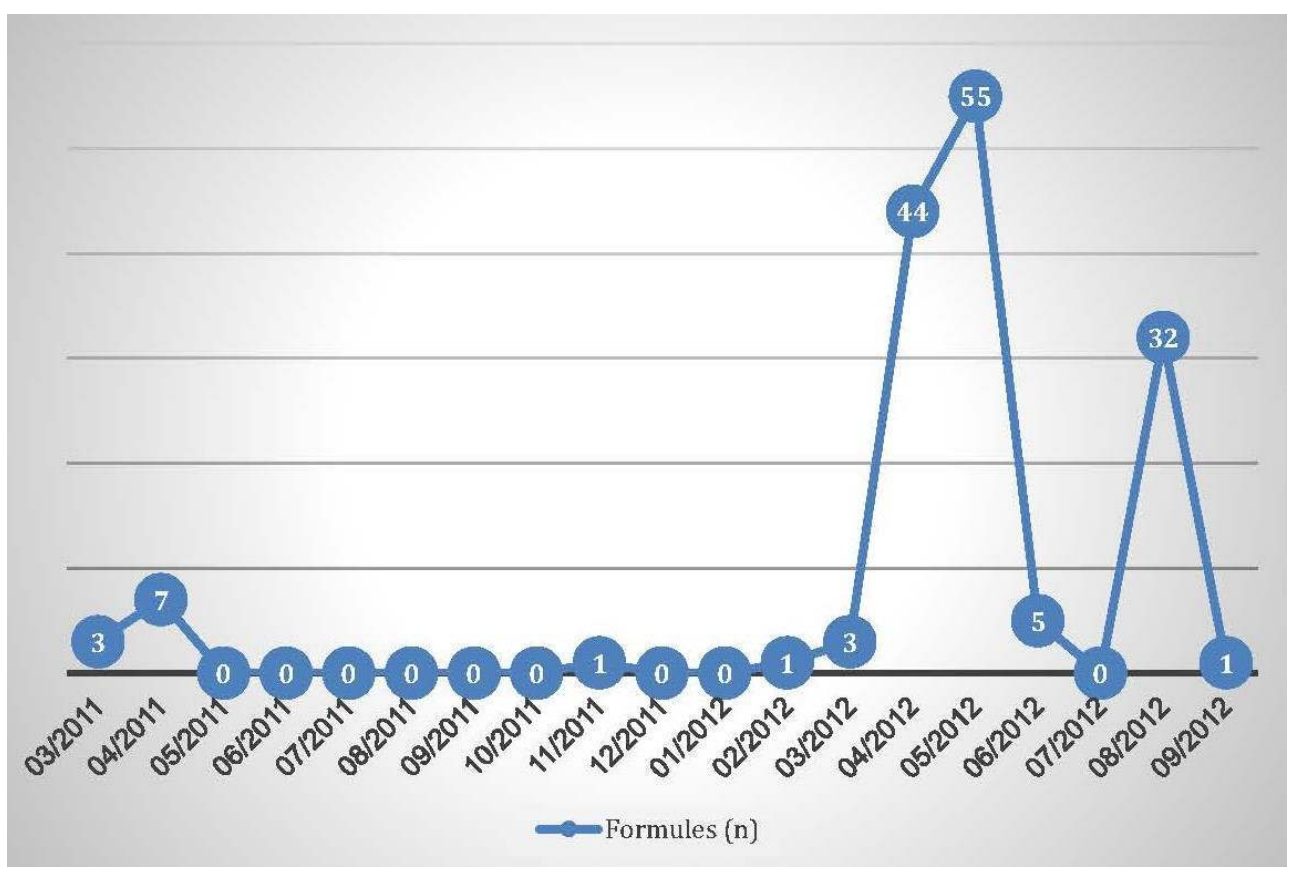

Figure 2 : Apparition des formules (n) selon le mois d'avril ( $\mathrm{n}=44)$, de mai $(\mathrm{n}=55)$, et d'août $(\mathrm{n}=32)$ 2012. Selon le rapport de la Commission spéciale d'examen des événements du printemps 2012, bien que le mouvement de "grève générale» débute au mois de février 2012, les négociations entre le gouvernement et les étudiants ne débutent qu’à la fin du mois de mars 2012. Ainsi, le mois d'avril est marqué par une «judiciarisation du conflit» (40) et une série de négociations entre le gouvernement et le mouvement étudiant, qui débouchent sur un nombre important de manifestations. Au mois de mai, les négociations et les manifestations se poursuivent, et le 4 mai 2012, elles culminent sur le plan de la violence $^{5}$ lors de la manifestation à Victoriaville, où a lieu le conseil général du PLQ. Le mois de mai marque aussi la démission de Line Beauchamp et l'arrivée de Michelle Courchesne au poste de ministre de l'Éducation ${ }^{6}$, le dépôt du projet de loi 78 intitulé «Loi permettant aux étudiants de recevoir l'enseignement dispensé par les établissements de niveau postsecondaire qu'ils fréquentent" (Commission spéciale d'examen des événements du printemps 2012, 2014), puis l'adoption de la loi qui en découle, la loi 12. Comme l'indique le rapport, « [l']été 2012 marque l'essoufflement des actions. La participation aux manifestations nocturnes est en baisse. On observe une diminution de la tension» $(2014: 103)$. Cela pourrait expliquer entre autres pourquoi très peu de formules - voire aucune - sont apparues durant les mois de juin $(n=5)$ et de juillet $(\mathrm{n}=0)$ en comparaison aux deux mois précédents (avril et mai) et au mois suivant (août). Par ailleurs, le $1^{\text {er }}$ août 2012, des élections générales sont déclenchées et les partis politiques impliqués entrent en campagne électorale, ce qui pourrait expliquer la montée observée pour ce mois $(\mathrm{n}=32)$. Somme toute, ces observations sur le plan de la distribution des formules dans le temps selon les mois et les événements du printemps érable confirment la présence d'un emploi accru de formules dans des moments forts de l'événement sociopolitique.

Des 152 formules recensées, le chef du gouvernement ${ }^{7}$ et la ministre Beauchamp de l'Éducation ont employé la très vaste majorité, soit respectivement $58,6 \%$ et 30,9\%. La 
ministre Courchesne de l'Éducation (5,3\%), le ministre des Finances (2,6\%) et le PLQ $(2,6 \%)$ ont, dans une moindre mesure, également fait appel à des formules pour s'exprimer dans le cadre du printemps érable. Il faut noter que Line Beauchamp a été ministre de l'Éducation du 11 août 2010 au 14 mai 2012, ce qui signifie que des 19 mois couverts par le corpus, elle a été en poste et en mesure de prendre la parole au nom du PLQ pendant 14 mois et demi. Michelle Courchesne ayant été désignée comme ministre remplaçante le 14 mai 2012, elle n'a pu être incluse que dans les articles publiés sur une période de quatre mois et demi.

Par ailleurs, les résultats indiquent que les deux principales entités visées par les formules sont le gouvernement lui-même $(41,4 \%)$ et les étudiants $(34,9 \%)$. Dans une moindre mesure, la chef de l'opposition (14,5\%), l'opposition officielle (5,3\%), et la population ou la société québécoise $(3,9 \%)$ ont également été la cible de formules employées par le gouvernement libéral. Ces résultats illustrent bien que le printemps érable a été caractérisé par une "guerre des mots" opposant le gouvernement aux associations étudiantes (Bonenfant 2013, Carbonneau et Desrochers 2013, Millette 2013, Poirier et Éthier 2013). Toutefois, ils mettent également en évidence trois autres entités impliquées dans le printemps érable et visées par la rhétorique du gouvernement, soient la chef de l'opposition, l'opposition officielle, et la population ou la société québécoise.

\subsection{Stratégies de cadrage sous-jacentes aux formules}

La Figure 3 illustre le nombre d'occurrences de chaque stratégie de cadrage pour les 152 formules recensées. Une même formule relève parfois de plusieurs stratégies de cadrage, ce qui a donné 322 occurrences de stratégies à analyser.

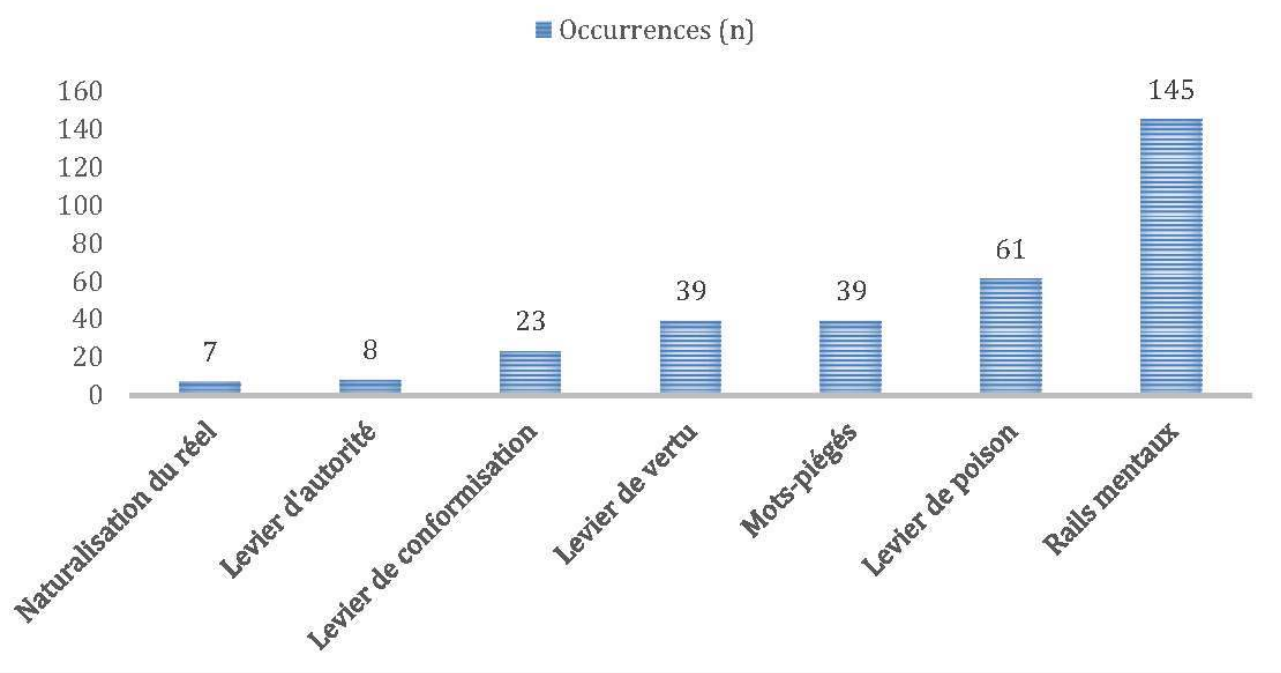

Figure 3 : Nombre d'occurrences selon la stratégie de cadrage

\subsubsection{Rails mentaux}

La stratégie de rails mentaux est la plus fréquente $(n=145)$. Cela pourrait s'expliquer entre autres par le fait que cette catégorie en recoupe plusieurs autres. En effet, elle regroupe toutes les formules qui envoient « notre pensée sur une tout autre voie que l'évaluation rationnelle » (Breton 1997, cité dans Carbonneau et Desrochers $2013: 8$ ) et 
qui proposent une nouvelle piste d'interprétation. À titre d'exemple, la formule «le gel des droits de scolarité, c'est une facture à payer $»^{8}$ illustre cette volonté de proposer une nouvelle piste d'interprétation pour le concept de gel de ces droits, afin de, l'on peut présumer, légitimer des choix politiques.

En ce qui concerne les locuteurs ayant utilisé cette stratégie, le chef du gouvernement affiche la plus grande proportion (60\%), suivi de la ministre Beauchamp de l'Éducation (29,7\%), puis du regroupement formé par la ministre Courchesne de l'Éducation, le ministre des Finances et le PLQ (10,3\%). Ces résultats sont à l'image des résultats généraux quant aux locuteurs.

Pour ce qui est de la cible des formules faisant appel aux rails mentaux, le discours peut chercher à cadrer l'image que le gouvernement projette :

- de lui-même (ex. : « faire tous les efforts possibles pour régler ce dossier $»^{9}$ )

- des étudiants (ex. : « fermer la porte à toute discussion ${ }^{10}$ )

- de la population québécoise (ex. : « les Québécois ne l'accepteront pas » ${ }^{11}$ )

- de l'opposition (ex. : « être silencieux quand il y a des actes de vandalisme $»^{12}$ )

- de la chef de l'opposition (ex. : « choisir d'être silencieuse ${ }^{13}$ )

D'un point de vue quantitatif, la plupart des rails mentaux visent le gouvernement $(40,7 \%)$ et les étudiants $(35,2 \%)$.

\subsubsection{Leviers poison}

La stratégie de leviers poison $(\mathrm{n}=61)$ vient au deuxième rang du nombre d'occurrences. Ces résultats font écho à ce qu'ont souligné Lacroix (2014) au sujet du « détournement de langage systématique » de la part du gouvernement : ceci aurait « mis en évidence la face guerrière de la langue de bois et sa violence symbolique » (233). Les leviers poison ont exclusivement été recensés dans des formules ciblant :

- les étudiants $(57,4 \%)$ ex. : « dénoncer la violence et l'intimidation $»^{14}$

- la chef de l'opposition (29,5\%) ex. : « abdication de leadership totale $»^{15}$

- l'opposition (13,1\%) ex. : «l'avenir du Québec n'est pas dans les moratoires et les gels $»^{16}$

32 Aucune formule ciblant le gouvernement ou la population n'a été catégorisée comme employant cette stratégie. En ayant recours à des formules qui définissent ou décrivent ses adversaires de façon défavorable, le gouvernement tente de faire accepter son idéologie et de délégitimer celle de ses opposants, sans doute pour conserver son statut d'entité dominante (Simpson 1993). Pour ce qui est de la population, on peut supposer que le gouvernement n'a aucun intérêt à l'associer à des formules ou des mots chargés négativement, s'il cherche à obtenir son appui.

\subsubsection{Leviers de vertu}

Le discours du gouvernement et les formules employées n'auraient pas seulement servi à remettre à sa place la minorité "privilégiée, radicale et violente " qu'aurait constituée le mouvement étudiant, comme l'ont avancé Sanschagrin et Gagnon $(2014: 260)$. En effet, on constate, en s'attardant aux formules ayant fait appel à la stratégie de leviers de vertu $(\mathrm{n}=39)$, que le gouvernement a voulu recadrer la réalité en la présentant sous un jour positif. En effet, les leviers de vertu sont principalement employés lorsque le gouvernement parle de lui-même ( $89,7 \%)$. Des formules telles que 
《toujours à l'écoute $»^{17}$, «saluer la patience, le courage et la détermination $»^{18}$ et " choix de la responsabilité ${ }^{19}$ sont employées afin de projeter une image positive du gouvernement, qui semble «vertueux » dans ses décisions et ses actions. À l'inverse, ce type de stratégie n'a pas du tout été observé lorsqu'il était question de l'opposition ou de la chef de l'opposition, et dans une plus faible proportion au sein de formules ciblant les étudiants $(7,7 \%)$ (ex. : «proposition porteuse de solutions $»^{20}$ ) et la population $(2,6 \%)$ (ex. : " principe d'équité entre les générations " $^{21}$ ). À cet effet, nous notons qu'il est intéressant que trois formules faisant appel aux leviers de vertu aient ciblé les étudiants, alors que la documentation insiste lourdement sur le mépris du gouvernement à leur égard (Ancelovici et Dupuis 2014, Bonenfant 2013, Julien 2012, Lacroix 2014, Lynes 2012, Millette 2013, Poirier et Éthier 2013, Sanschagrin et Gagnon 2014, Sorochan 2012). Par ailleurs, nous pourrions nous attendre à ce que la stratégie de leviers de vertu soit employée plus largement pour cibler la population ou la société en général, le gouvernement cherchant à gagner son appui, mais nos résultats n'indiquent en rien cette tendance.

\subsubsection{Mots piégés}

Pour ce qui est des mots piégés ( $n=39$ ), ces « "vocables au contenu tendancieux", qui par leur simple usage suscitent l'indignation» (Carbonneau et Desrochers 2013:8), nous avons observé que les formules y faisant appel s'appuyaient également sur une ou plusieurs autres stratégies de cadrage. Autrement dit, cette stratégie de cadrage n'a pas été employée seule ; en fait, dans les 39 occurrences où elle a été identifiée, elle était associée à la stratégie de rails mentaux. À l'instar de Carbonneau et Desrochers, nous avons aussi constaté que les mots piégés étaient des mots « $a$ priori négativement chargés» (8), ce qui explique que les formules classées dans cette catégorie apparaissaient en concomitance avec la stratégie de leviers poison. Les résultats sont également probants lorsqu'on observe la cible des formules. De façon analogue aux leviers poison, la stratégie de mots piégés a surtout été mise à profit dans le cas de formules visant les adversaires du gouvernement :

- les étudiants ( $48,7 \%)$ ex. : « la rue a fait beaucoup de bruit $»^{22}$

- la chef de l'opposition $(35,9 \%)$ ex. : « se comporter de façon indéfendable " ${ }^{23}$

- l'opposition $(12,8 \%)$ ex. : «choisir un gouvernement péquiste dont les gestes et décisions sont dictés par la rue $»^{24}$

Comme l'ont souligné Carbonneau et Desrochers, les mots piégés sont « d'autant plus péjorativement chargés qu'ils ne sont pas utilisés dans le contexte du phénomène qu'ils désignent sémantiquement » (8). Dans l'exemple «la rue a fait beaucoup de bruit », le mot « rue » ne désigne pas sémantiquement l'objet réel, mais fait référence à un groupe social que le gouvernement tente de délégitimer en l'associant à un symbole négatif. Similairement, en employant les formules «se comporter de façon indéfendable » et " choisir un gouvernement péquiste dont les gestes et décisions sont dictés par la rue ", le gouvernement cherche à susciter l'indignation de tous vis-à-vis des comportements de ses adversaires politiques, à savoir la chef de l'opposition et le parti qu'elle représente. 


\subsubsection{Leviers de conformisation} sont :

- le gouvernement lui-même (50 \%) ex. : « choisir un gouvernement qui fait respecter la loi et l'ordre ${ }^{33}$

- les étudiants $(37,5 \%)$ ex. : « le non-respect des lois, ce n'est pas responsable $»^{34}$

- la chef de l'opposition (12,5\%) ex. : « choisir la rue au lieu de la démocratie $»^{35}$

Les leviers de conformisation ( $\mathrm{n}=23)$ visent à rallier le public aux propos illustrés par la formule. La formule "majorité silencieuse $»^{25}$ illustre bien cette stratégie : le locuteur cherche à associer le destinataire à une "communauté humaine en particulier" (Carbonneau et Desrochers $2013: 9$ ), que celle-ci existe ou non. Les résultats pour cette stratégie de cadrage affichent des proportions similaires pour ce qui est de la cible des formules, soit :

- le gouvernement $(30,4 \%)$ ex. : « c'est le camp de la solution [ou de la perturbation] $»^{26}$ - les étudiants $(26,1 \%)$ ex. : « la violence est inacceptable pour la société québécoise " ${ }^{27}$ - la société québécoise $(26,1 \%)$ ex. : « Comme moi, la population dit assez, c'est assez ! » 28

Cette volonté d'appeler à la solidarité semble donc être une stratégie qui s'emploie de façon générique, que le gouvernement emploie des formules pour cibler certains adversaires, la population en général ou lui-même. Par contre, pour ce qui est de la chef de l'opposition et de l'opposition, les proportions sont plus faibles ( $8,7 \%$ dans les deux cas).

la population, il interpelle aussi la société québécoise, mais ultimement pour parler des étudiants lorsqu'il emploie la formule « des gestes [de violence] qui ont étonné tous les Québécois $»^{30}$.

\subsubsection{Leviers d'autorité} une moindre mesure $(n=8)$. Les formules faisant appel aux leviers d'autorité misent sur la voix de l'expérience, sur l'avis de figures d'autorité ou d'institutions, afin de cadrer le message véhiculé (Breton 1997). C'est le cas de la formule «choisir la rue au lieu de la démocratie $»^{31}$, par laquelle l'interlocuteur - en l'occurrence, le chef du gouvernement - s'appuie sur l'institution et fait appel au concept de la démocratie pour faire référence à un choix qui se présente au public. Le problème étant ainsi cadré, le public a le choix de s'associer à un principe relevant de l'autorité de la démocratie, ou à la rue, désignée comme le "camp » des étudiants. Le chef du gouvernement est celui ayant le plus employé de formules $(87,5 \%)$ faisant appel aux leviers d'autorité (ex. : " contester l'ordre établi et nos institutions ${ }^{32}$ ). Il s'agit de la stratégie pour laquelle il est le plus souvent cité. Il est difficile de ne pas faire de lien entre le concept d'autorité et son incarnation: il semble logique que la figure d'autorité, celle ultimement responsable de la rhétorique du groupe, soit celle qui évoque le plus le concept d'autorité, appuyant ses idées et idéologies sur celui-ci (Simpson 1993).

Pour ce qui est des cibles des formules faisant appel aux leviers d'autorité, celles-ci Le fait de s'appuyer sur une figure d'autorité ou sur des institutions sociales comme stratégie de cadrage a servi au gouvernement pour cadrer les propos qu'il tenait sur 
lui-même et pour critiquer les idées de ses adversaires. Nous notons qu'aucune formule ciblant la population ou l'opposition n'a employé les leviers d'autorité.

\subsubsection{Naturalisation du réel}

Enfin, les formules qui font appel à la naturalisation du réel $(n=7)$ sont factuelles, non connotées positivement ou négativement, et servent à déresponsabiliser ou sursimplifier les propos ou les idées de l'interlocuteur. Par exemple, avec la formule "hausse de 50 cents par jour ${ }^{36}$, l'interprétation de la réalité suggérée n'est pas éloignée de la rationalité - au contraire -, mais elle sert à simplifier le message et à le rendre percutant et "calculable». Alors que les autres stratégies étaient majoritairement employées au sein de formules prononcées par le chef du gouvernement (60 à 87,5\%), le cas de figure diffère grandement pour la naturalisation du réel: c'est la ministre Beauchamp de l'Éducation qui a le plus employé cette stratégie $(71,4 \%)$. Il convient cependant de rappeler encore une fois que le nombre de formules recourant à cette stratégie n'est que de sept. Néanmoins, selon les résultats obtenus, la stratégie de cette locutrice semble s'être essentiellement articulée autour de la rationalisation du message (ex.: «c'est zéro, l'endettement $»^{37}$, «hausse de 50 sous par jour $\aleph^{38}$, «trêve de 48 heures $»^{39}$ ). Elle ne semble pas avoir eu le même mandat que son chef de parti d'utiliser des formules ciblant l'opposition et la chef de l'opposition. Selon les rôles différenciés des politiciens prenant part au discours du gouvernement, des stratégies différentes sont certainement envisagées, planifiées et mises en application.

\section{Conclusion}

Dans cet article, nous avons analysé 152 formules employées par le gouvernement pendant le printemps érable, une crise sociopolitique qui a marqué le Québec en 2012. L'analyse a révélé plusieurs aspects qui méritent d'être rappelés :

- Une utilisation accrue de formules a été observée pendant les mois cruciaux du printemps érable (avril et mai 2012) et le mois d'août 2012.

- La majorité des formules avaient pour cibles le gouvernement lui-même et les étudiants. Trois autres entités ont été relevées, soient la chef de l'opposition, l'opposition politique, ainsi que la société civile québécoise.

- Cinq locuteurs ayant employé des formules ont été identifiés: le chef du gouvernement, les ministres de l'Éducation, le ministre des Finances et le PLQ. Le chef du gouvernement était le principal utilisateur de formules.

- Les stratégies de cadrage sous-tendant les formules employées par le gouvernement dénotent des nuances sur le plan de la rhétorique du gouvernement ; cette dernière n'apparait pas aussi homogène que ce qu'avaient suggéré les sources consultées.

Tout compte fait, la représentation du printemps érable comme opposant le gouvernement au mouvement étudiant est confirmée mais doit être nuancée. Bien que ces deux entités aient majoritairement été ciblées par les formules, l'analyse a révélé d'autres dimensions, notamment la présence de multiples locuteurs et de cibles s'inscrivant dans la stratégie discursive. Le discours du gouvernement et les formules employées n'auraient donc pas seulement servi à "délégitimer " les étudiants, mais aussi à cadrer l'image du gouvernement lui-même et celle de ses adversaires politiques. 
Les formules du gouvernement seraient donc des traces discursives des efforts de ce dernier à réfuter les arguments du mouvement étudiant et à renouveler constamment ses propres arguments. Comme l'explique Orkibi (2015), le discours d'un groupe suscite le discours des adversaires, et vice versa.

Le concept de formule aura permis de produire des unités d'analyse pouvant être étudiées empiriquement au regard du cadrage et de diversifier les recherches menées sur l'événement sociodiscursif qu'est le printemps érable.

Sur le plan méthodologique, bien que les articles de quotidiens bénéficient d'un statut privilégié en analyse du discours, la diffusion du discours et des formules qui le forment s'est exécutée simultanément dans une multitude de médias: réseaux sociaux, télévision, radio et sites web constituent quelques exemples de médias qui pourraient être mis à contribution. En outre, les stratégies, les cibles, les locuteurs et toute autre variable varient-ils en fonction du média exploité?

Lors de la collecte des données, plusieurs formules similaires ou identiques à celles employées par le gouvernement ont surgi dans le discours de leaders étudiants et de membres de l'opposition, par exemple «mettre le feu aux poudres $»^{40}$, «sortir de la crise $»^{41}$, « détourner le débat du vrai enjeu ${ }^{42}$, «calmer la grogne des étudiants $»^{43}$, "discuter dans le calme ${ }^{44}$ et "perdre toute légitimité et crédibilité »" Dès lors, il semble pertinent de s'intéresser au discours de l'entité dominante, tel que proposé par le courant de l'analyse critique du discours (Fairclough 2001, Simpson 1993, Van Dijk 1985) non seulement dans les discours où elle est identifiée comme locuteur, mais également dans le discours des entités adverses (par exemple des étudiants et de l'opposition). Le caractère non exclusif de certaines des stratégies et des formules employées par le gouvernement et certains représentants du mouvement étudiant ou de l'opposition permet de mieux saisir la complexité du discours social et de comprendre comment sa circulation influence tous les acteurs. L'identification de similitudes quant aux éléments discursifs des différents acteurs permettrait de dépasser ce qui semble être un clivage hâtif : le gouvernement est-il vraiment si retors et les opposants sont-ils si transparents sur le plan des communications publiques?

Des études de la sorte permettent non seulement de mettre en évidence certains aspects idéologiques qui sous-tendent les discours d'entités dominantes et de clarifier les liens entre le langage et les relations de pouvoir, mais aussi, et ultimement, de conscientiser le public aux pressions auxquelles il est exposé.

\section{BIBLIOGRAPHIE}

Ancelovici, Marcos \& Francis Dupuis-Déri. 2014. «Introduction : retour sur le "Printemps érable" ", Ancelovici, Marcos \& Francis Dupuis-Déri (éds.). Un printemps rouge et noir : regards croisés sur la grève étudiante de 2012 (Montréal : Éditions Écosociété), 7-36

Asselin, Olivier. 2012, « Carré rouge. Le destin politique d'une forme colorée », Theory \& Event [En ligne] $15: 3$, http://muse.jhu.edu/journals/theory_and_event/v015/15.3S.asselin.html 
Bégin-Caouette, Olivier \& Glen A. Jones. 2014. «Student organizations in Canada and Quebec's 'Maple Spring' ", Studies in Higher Education 39(3), 412-425

Benford, Robert \& David Snow. 2000. « Framing processes and social movements : an overview and assessment », Annual Review of Sociology 26, 611-639

Bernard-Barbeau, Geneviève. 2015. «De l'appel à mobilisation à ses mécanismes sociodiscursifs : le cas des slogans écrits du printemps érable », Argumentation et analyse du discours 14, https:// journals.openedition.org/aad/1969

Bonenfant, Maude, Anthony Glinoer \& Martine-Emmanuelle Lapointe. 2013. Le printemps québécois : une anthologie (Montréal : Éditions Écosociété)

Breton, Philippe. 1997. La parole manipulée (Montréal : Boréale)

Carbonneau, Jean-Rémi \& Mélissa Desrochers. 2013. Réflexion sur le rôle et la polarisation des médias dans le cadre du conflit étudiant québécois 2012. Communication présentée au Congrès annuel de l'Association canadienne de science politique, [En ligne] http://www.cpsa-acsp.ca/papers-2013/ Carbonneau.pdf

Collette, Karine 2013. « Distances et conciliations sur le statut du discours : autour d'une analyse émergente des discours du Printemps érable ", Cahiers de recherche sociologique 54, 71-94

Commission spéciale d'examen des événements du printemps 2012. Rapport-Commission spéciale d'examen des événements du printemps 2012, [En ligne] https://www.securitepublique.gouv.qc.ca/ fileadmin/Documents/police/publications/rapport_CSEEP2012/rapport_CSEP2012.pdf

Entman, Robert M. 1993. « Framing: toward clarification of a fractured paradigm », Journal of Communication $43: 4,51-58$

Fairclough, Norman. 2001. Language and power (Harlow: Longman)

Frappier, André, Richard Poulin \& Bernard Rioux. 2012. Le printemps des carrés rouges : lutte étudiante, crise sociale, loi liberticide, démocratie de la rue (Montréal : M éditeur)

Gauthier, Gilles. 2016. «Le "printemps érable” au Québec : “Grève” ou "boycott”? Les enjeux stratégiques d'un conflit de nomination », Argumentation et analyse du discours 17, https:// journals.openedition.org/aad/2248

Julien, Frédéric. 2012. « Le printemps érable comme choc idéologique », Cultures \& Conflits 3, 152-159

Krieg-Planque, Alice. 2009. La notion de « formule » en analyse du discours : cadre théorique et méthodologique (Besançon : P. U. de Franche-Comte)

Lacroix, Michel, Rachel Nadon \& Oliver Parenteau. 2014. « La grève en vers et en prose : combats, silences et fissures », Ancelovici, Marcos \& Francis Dupuis-Déri (éds.). Un printemps rouge et noir : regards croisés sur la grève étudiante de 2012 (Montréal : Éditions Écosociété), 233-255

Lynes, Krista Geneviève. 2012. "Poetic resistance and the classroom without guarantees ", Theory \& Event [En ligne] $15: 3$, http://muse.jhu.edu/journals/theory_and_event/v015/15.3S.lynes.html Millette, Josianne. 2013. « Chapitre 4 : Communiquer pour gagner ", Josianne. Millette (éd.). De la rue au fil de presse : grèves étudiantes et relations publiques (Québec: P. U. Laval), 61-89

Orkibi, Eithan. 2015. « Le(s) discours de l'action collective : contextes, dynamiques et traditions de recherche ", Argumentation et Analyse du Discours 14, https://journals.openedition.org/aad/ 2002 
Poirier St-Pierre, Renaud \& Philippe Éthier. 2013. De l'école à la rue : dans les coulisses de la grève étudiante (Montréal : Éditions Écosociété)

Sanschagrin, David \& Alain-G. Gagnon. 2014. «L'approfondissement du politique au Québec. Les partis politiques et la grève étudiante de 2012 ", Marcos Ancelovici \& Francis Dupuis-Déri (éds.). Un printemps rouge et noir : regards croisés sur la grève étudiante de 2012 (Montréal : Éditions Écosociété), 259-294

Simpson, Paul. 1993. Language, ideology and point of view (New York: Routledge) Sorochan, Cayley. 2012. «The Quebec student strike - A chronology », Theory \& Event [En ligne] $15: 3$, http://muse.jhu.edu/journals/theory_and_event/v015/15.3S.sorochan.html.

Van Dijk, Teun A. 1985. Handbook of discourse analysis, 4 vol. (Londres: Academic Press)

\section{ANNEXES}

\section{Liste des articles du corpus cités et numérotés}

Article 1

AGENCE QMI. (2012, $1^{\mathrm{er}}$ mai). « Line Beauchamp inflexible ». 24 heures. Récupéré de : http://mandataire.uqo.ca:2738/Search/ResultMobile/0, consulté en ligne dans la base de données Eureka le 2 septembre 2017.

Article 2

AGENCE QMI (2012, 2 août). « Jean Charest fera campagne sur le conflit étudiant ». 24 heures. Récupéré de : http://mandataire.uqo.ca:2738/Search/ResultMobile/2, consulté en ligne dans la base de données Eureka le 2 septembre 2017.

Article 3

Breton, P. (2012, 29 mars). « Guerre d'usure ». La Presse. Récupéré de : http:// proxybiblio.uqo.ca:2529/Search/ResultMobile/3, consulté en ligne dans la base de données Eureka le 2 septembre.

Article 4

Shields, A. (2012, 21 avril). « Émeute à l'extérieur, sarcasme à l'intérieur ». Le Devoir. Récupéré de : http://proxybiblio.uqo.ca:2529/Search/ResultMobile/0, consulté en ligne dans la base de données Eureka le 2 septembre.

Article 5

Lavallée, J.-L. (2012, 14 avril). « Une "faute impardonnable" ». Journal de Montréal. Récupéré de : http://mandataire.uqo.ca:2738/Search/ResultMobile/2, consulté en ligne dans la base de données Eureka le 2 septembre 2017.

Article 6

Elkouri, R. (2012, 24 septembre). « Les cicatrices du printemps ». La Presse. Récupéré de : http://mandataire.uqo.ca:2738/Search/ResultMobile/2, consulté en ligne dans la base de données Eureka le 2 septembre 2017.

Article 7

Marquis, M. (2011, 4 avril). « Manifestation d'étudiants au colloque régional du Parti libéral du Québec - Les libéraux demeurent intraitables sur la hausse des droits de scolarité ». Le Devoir. Récupéré de : http://proxybiblio.uqo.ca:2529/Search/ ResultMobile, consulté en ligne dans la base de données Eureka le 2 septembre. Article 8

AGENCE QMI. (2012, 15 mai). « Line Beauchamp passe le flambeau à Michelle 
Courchesne ». 24 heures. Récupéré de : http://mandataire.uqo.ca:2738/Search/

ResultMobile/0, consulté en ligne dans la base de données Eureka le 2 septembre 2017. Article 9

Dutrisac, R. (2012, 23 juin). « Jean Charest pris entre deux feux ». Le Devoir. Récupéré de : http://proxybiblio.uqo.ca:2529/Search/ResultMobile/0, consulté en ligne dans la base de données Eureka le 2 septembre 2017.

Article 10

Teisceira-Lessard, P. (2012, 16 avril). « Une ouverture, des conditions ». La Presse. Récupéré de : http://proxybiblio.uqo.ca:2529/Search/ResultMobile/0, consulté en ligne dans la base de données Eureka le 2 septembre 2017.

Article 11

Ménard, S. (2011, 18 mars). « 325 \$ de plus par année ». Journal de Montréal. Récupéré de : http://proxybiblio.uqo.ca:2529/Search/ResultMobile/0, consulté en ligne dans la base de données Eureka le 2 septembre 2017.

Article 12

Nadeau, R. (2012, 7 mai). « Jean Charest ne regrette rien ». Journal de Montréal. Récupéré de : http://proxybiblio.uqo.ca:2529/Search/ResultMobile/0, consulté en ligne dans la base de données Eureka le 2 septembre 2017.

Article 13

Chouinard, T. (2012, 24 avril). «Beauchamp ouvre la porte ». La Presse. Récupéré de : http://proxybiblio.uqo.ca:2529/Search/ResultMobile/0, consulté en ligne dans la base de données Eureka le 2 septembre 2017.

Article 14

Lavallée, J.-L. (2012, 28 avril). « Une hausse étalée sur 7 ans ». Journal de Montréal. Récupéré de : http://proxybiblio.uqo.ca:2529/Search/ResultMobile/0, consulté en ligne dans la base de données Eureka le 2 septembre 2017.

Article 15

Breton, P. et Chouinard, T. (2012, 8 mai). « Les étudiants disent non ». La Presse.

Récupéré de : http://proxybiblio.uqo.ca:2529/Search/ResultMobile/0, consulté en ligne dans la base de données Eureka le 2 septembre 2017.

Article 16

Lefebvre, S.-M. (2012, 6 avril). « Les étudiants se sentent incompris ». Journal de Montréal. Récupéré de : http://proxybiblio.uqo.ca:2529/Search/ResultMobile/0, consulté en ligne dans la base de données Eureka le 2 septembre 2017.

\section{NOTES}

1. Dans son ouvrage, l'auteure couvre deux événements : la grève étudiante de 2005 et celle de 2012. Pour l'année qui nous intéresse, 2012, elle a mené sept entrevues avec « des personnes ayant été membres des comités exécutifs ou des comités Médias de la CLASSÉ, de la FEUQ et de la FECQ ainsi qu'avec une personne affiliée à Force étudiante critique » (160).

2. L'emploi de l'italique provient de la source originale.

3. Breton cite ici S. Tchakhotine. 1952. Le Viol des foules par la propagande politique (Paris: Gallimard)

4. Le 17 mars 2011 marque le dépôt du plan budgétaire du gouvernement Charest annonçant la hausse des frais de scolarité ; le 4 septembre 2012 est la date à laquelle le Parti québécois remporte les élections provinciales (Commission spéciale d'examen des événements du printemps 2012, 2014). 
5. À cet effet, le rapport souligne que « La manifestation du 4 mai est considérée comme l'une des plus violentes depuis la tenue du Sommet des Amériques en $2001 »$ (2014: 77).

6. Afin de simplifier la structure phrastique, nous utilisons l'abréviation Éducation pour référer au ministère de l'Éducation, du Loisir et du Sport.

7. Soulignons d'emblée que deux catégories distinctes ont été créées pour rendre compte du fait que parfois, le locuteur ayant employé une formule pouvait constituer un individu (en l'occurrence, le chef du gouvernement, la ministre de l'Éducation ou le ministre des Finances) ou un groupe d'individus (en l'occurrence, le Parti libéral du Québec).

8. Article 1: Afin d'alléger la présentation des notes de bas de page, nous avons attribué un numéro à chaque article du corpus cité. La liste des articles numérotés se trouve en annexe.

9. Article 2

10. Article 3

11. Article 4

12. Article 5

13. Article 5

14. Article 6

15. Article 5

16. Article 4

17. Article 7

18. Article 8

19. Article 9

20. Article 10

21. Article 11

22. Article 2

23. Article 12

24. Article 2

25. Article 2

26. Article 13

27. Article 4

28. Article 8

29. Article 2

30. Article 12

31. Article 5

32. Article 2

33. Article 2

34. Article 13

35. Article 5

36. Article 14

37. Article 11

38. Article 14

39. Article 13

40. Gabriel Nadeau-Dubois (CLASSÉ) dans l'article 15

41. ibid.

42. Martine Desjardins (FEUQ) dans l'article 16

43. Léo Bureau-Blouin (FECQ) dans l'article 16

44. Marie Malavoy (PQ) dans l'article 5

45. ibid. 


\section{RÉSUMÉS}

Notre recherche porte sur la stratégie discursive du gouvernement pendant le printemps érable québécois de 2012, particulièrement sur les formules qu'il a employées afin d'influencer l'opinion publique. Au moyen d'une analyse de contenu de 100 articles de cinq quotidiens québécois, nous avons étudié 152 formules au regard de leur distribution dans le temps, des entités qu'elles ont ciblées, des locuteurs les ayant employées et des stratégies de cadrage qu'elles sous-entendent. L'analyse révèle plusieurs aspects, notamment la présence de multiples locuteurs et de cibles s'inscrivant dans la stratégie discursive. Le discours et les formules employées ont cherché à délégitimer les étudiants, mais aussi à cadrer l'image du gouvernement lui-même et celle de ses adversaires politiques. Le concept de formule permet de produire des unités d'analyse pouvant être étudiées empiriquement au regard du cadrage et de diversifier les recherches menées sur l'événement sociodiscursif qu'est le printemps érable.

Our research focuses on the government's discursive strategy during the 2012 Maple Spring in Quebec, particularly on the formulas used to influence public opinion. Through a content analysis of 100 articles from five Quebec newspapers, we studied 152 formulas with regard to their distribution in time, the entities they targeted, the speakers who used them, and the framing strategies they conveyed. The analysis revealed several aspects, including the presence of multiple speakers and targets as part of the discursive strategy. The discourse and the formulas that were used sought to delegitimize the students, but also to frame the image of the government itself, and that of its political opponents. Eventually, the concept of formula allowed us to produce units of analysis that could be studied empirically with regard to framing, and to contribute to the analysis of the Maple Spring as a socio-discursive event.

\section{INDEX}

Mots-clés : cadrage, formule printemps érable

Keywords : formula, framing, Maple Spring

\section{AUTEURS}

\section{LAURENCE PELLETIER}

Université du Québec en Outaouais

MARIE-JOSÉE GOULET

Université du Québec en Outaouais 